\title{
Fenotipeo y selección de líneas S1 segregantes de maíz tolerantes a estrés hídrico
}

\author{
Phenotyping and selection of $\mathrm{S}_{1}$ segregant inbred maize lines tolerant to hydric stress
}

\begin{abstract}
Hermes Rebolloza-Hernández ${ }^{1 *}$, Yessica F. Cervantes-Adame', Elizabeth Broa-Rojas², Gregorio Bahena-Delgado', Angeluz Olvera-Velona'

Escuela de Estudios Superiores de Xalostoc, Universidad Autónoma del Estado de Morelos (UAEM), Avenida Nicolás Bravo S/N, Parque Industrial Cuautla, Cd. Ayala, Morelos, México, C. P. 62715. Tel. (777) 3297000 Ext. 7981.

2 Escuela de Estudios Superiores de Totolapan, Universidad Autónoma del Estado de Morelos (UAEM), Carretera TotolapanNepopoalco S/N, Ahuatlán, Totolapan, Morelos, México, C. P. 62830. Tel. (735) 3579977.
\end{abstract}

\section{RESUMEN}

El maíz es susceptible a la sequía, una estrategia de solución es generar líneas tolerantes a partir de una población segregante para obtener híbridos o variedades sintéticas, por ello los objetivos fueron seleccionar líneas de rendimiento alto y ASI corto con base al sistema riego-sequía, estimar la heredabilidad y la correlación de caracteres cuantitativos. El cruzamiento de las líneas Ac7643 y B39 derivó 193 líneas $S_{1^{\prime}}$ estas líneas junto con el testigo y progenitores se evaluaron bajo el diseño alfa látice en un arreglo $14 \times 14$ con dos repeticiones en dos localidades de Morelos, México. Las variables fueron floración masculina (FM) y femenina (FF), intervalo antesis-emergencia de estigmas (ASI), altura de planta (AP) y mazorca (AM); y rendimiento de grano (RG). Se observó reducción del $40 \%$ en RG bajo sequía ( $\left.0.75 \mathrm{t} \mathrm{ha}^{-1}\right)$ con respecto a riego ( 1.26 ton ha-1), el ASI fue de 5 y $2 \mathrm{~d}$ en sequía y en riego, respectivamente. La heredabilidad para RG y ASI fue de 0.41 . Existen líneas con herencia transgresiva para RG y ASI. La correlación del RG con FF y ASI fue de $-0.23^{* *} y-0.37^{* *}$, respectivamente. El germoplasma puede generar una variedad sintética o híbridos tolerantes a sequía.

Palabras clave: Caracterización, ASI, rendimiento de grano.

\section{ABSTRACT}

Corn is susceptible to drought; a solution strategy is to generate tolerant lines from a segregating population to obtain hybrids or synthetic varieties, so the objectives were to select high yield and short ASI lines based on the irrigation-drought system, to estimate heritability and correlation of quantitative traits. The crossing of the Ac7643 × B39 lines, derived in $193 \mathrm{~S}_{1}$ lines which, together with witness and parents, were evaluated under the alpha-lattice design in a $14 \times 14$ arrangement with two repetitions in two locations in Morelos, Mexico. The variables were flowering male (MF) and female (FF), anthesis-silking interval (ASI), plant height (HP) and cob (HC), as well as grain yield (GY). We observed a $40 \%$ reduction in $\mathrm{GY}$ under drought $\left(0.75 \mathrm{t} \mathrm{ha}^{-1}\right)$ with respect to the irrigation environment $\left(1.26 \mathrm{t} \mathrm{ha}^{-1}\right)$. The ASI was five and two days in drought and irrigation environments, respectively. The heritability for GY and ASI was 0.41. There are lines with transgressive inheritance for GY and ASI. The correlation of the GY with FF and ASI was $-0.23 * *$ and $-0.37^{* *}$, respectively.

Volumen XXII, Número 3
The germplasm can generate a synthetic variety or drought tolerant hybrids.

Keywords: Characterization, ASI, grain yield.

\section{INTRODUCCIÓN}

Los rendimientos bajos de grano de maíz tropical y los impactos del cambio climático, específicamente el incremento de la temperatura en las zonas propensas a estrés hídrico, priorizan la necesidad de realizar mejoramiento genético para incrementar el rendimiento de grano de maíz (Cairns et al., 2013). Las pérdidas en la producción mundial de este cereal a causa de la sequía es del $21 \%$ (Edmeades, 2013; Xue et al., 2013), y dicha reducción se incrementa al $39.3 \%$ cuando el déficit hídrico es intenso; por lo que el maíz es susceptible a la sequía en la etapa fenológica previa y durante la fase reproductiva (Daryanto et al., 2016).

Las zonas maiceras y la productividad de los agroecosistemas que se ubican en zonas tropicales están sujetas a la variación aleatoria en distribución y cantidad de la precipitación pluvial. México no está exento de esta condición ambiental y los impactos negativos se reflejan en los rendimientos de grano de maíz bajo temporal (83\% de la superficie sembrada en México) con rendimiento promedio de los últimos cinco años de $3.2 \mathrm{t} \mathrm{ha}^{-1}$ (SAGARPA-SIAP, 2018).

El mejoramiento genético para tolerancia a sequía puede subsanar hasta el $25 \%$ las pérdidas de rendimiento de grano. En este contexto, el Centro Internacional de Mejoramiento de Maíz y Trigo (CIMMYT) realiza selección convencional para tolerancia a sequía y generó un incremento de $100 \mathrm{~kg} \mathrm{ha}^{-1} \mathrm{a}^{-1}$ en rendimiento de grano en poblaciones de maíz tropical a través de caracteres asociados al estrés hídrico (Edmeades, 2013). En este orden, las estrategias de mejoramiento genético para tolerancia a la sequía requieren del fenotipeo de progenies endogámicas, las cuales pueden ser útiles para optimizar la eficiencia del mejoramiento genético convencional para el estrés hídrico en maíz. Por lo tanto, las líneas bajo estrés por sequía se pueden utilizar para desarrollar híbridos para ambientes severamente propensos a este factor de tensión (Kebede et al., 2013). En esta perspectiva, la obtención de líneas $S_{1}$ se realiza a partir de una población $\mathrm{F}_{2^{\prime}}$ en la cual la varianza genotípica se incrementa en relación a la generación filial $F_{1}$ debido a la segregación

*Autor para correspondencia: Hermes Rebolloza Hernández Correo electrónico: hermes.rebollozah@uaem.edu.mx 
alélica (Walsh, 2001). Una vez que se tienen las poblaciones segregantes en maíz, se derivan líneas endogámicas y se puede establecer diseños de apareamiento genético para determinar los patrones heteróticos al que pertenecen el grupo de líneas y obtener híbridos sobresalientes (González et al., 1997); o bien, dentro de un grupo heterótico seleccionar líneas sobresalientes y generar variedades sintéticas (Terrón et al., 1997). En este contexto, existe el reporte de estudios que identificaron líneas endogámicas con alta tolerancia a la sequía y el uso potencial de estas líneas puede generar híbridos de maíz que sean capaces de mitigar los impactos negativos del estrés hídrico en el crecimiento y desarrollo de plantas de maíz (Chen et al., 2012). La buena funcionalidad de híbridos en maíz tropical tolerantes a estrés hídrico se puede lograr a través de manipular artificialmente los niveles de humedad (Betrán et al., 2003). En adición a lo anterior, el intervalo entre Antesis Emergencia de estigmas (ASI) se usa como un indicador fenotípico eficiente para tolerancia a sequía y también se utiliza en programas de mejoramiento para incrementar la estabilidad del rendimiento bajo condiciones de estrés hídrico (Machado et al., 2002). Asimismo, el carácter de transpiración limitada puede ser útil en maíz para incrementar el rendimiento en ambientes propensos a sequía ya que el impacto del carácter en la producción del maíz varía en función a la geografía, ambiente, expresión del carácter y densidad poblacional (Messina et al., 2015).

La selección de líneas tolerantes a estrés hídrico se debe realizar con base a ambientes contrastantes de humedad para generar variedades sintéticas o híbridos (Veldboom y Lee, 1996; Kebede et al., 2013); en esta perspectiva, los objetivos en esta investigación fueron los siguientes: 1) fenotipear a la población segregante de maíz en ambientes limitantes y no limitantes de humedad, 2) seleccionar líneas $\mathrm{S}$ de alto rendimiento de grano y ASI corto bajo condiciones de estrés hídrico y buen comportamiento agronómico en riego y 3) determinar la heredabilidad y correlación de los caracteres cuantitativos.

\section{MATERIALES Y MÉTODOS}

El germoplasma que se utilizó en esta investigación fue 193 líneas $S_{1}$, las líneas parentales Ac7643 y B39 (progenitor femenino y masculino, respectivamente); y una línea endogámica tolerante a estrés hídrico como testigo (Ac7729/ TZSRW). La línea Ac7643 es germoplasma tropical y se derivó de la Población 43 (La Posta Sequía) bajo el criterio de selección para tolerancia al estrés hídrico, dicha línea se usó en diferentes estudios para tolerancia a la sequía (Ribaut et al., 1996 y 1997); mientras que la línea B39 provino del INIFAP de Celaya, Guanajuato, México; ésta se derivó con criterio principal de selección para aptitud combinatoria general y es progenitor de los híbridos comerciales de maíz de grano blanco H-358 y H-359 (Ramírez et al., 1995).

La cruza directa de Ac7643 x B39 se realizó en el ciclo primavera-verano 2010, en el campo experimental de la Escuela de Estudios Superiores de Xalostoc (EESuX), depen- diente de la Universidad Autónoma del Estado de Morelos. En esta misma localidad, durante primavera-verano 2012, se sembró la generación $\mathrm{F}_{1}$ en un lote de $500 \mathrm{~m}^{2}$ y al momento de la floración se realizó la autofecundación de 120 plantas. Posteriormente, durante la cosecha se eligieron 80 mazorcas con base en sanidad, las cuales se desgranaron y mezclaron para formar la población $\mathrm{F}_{2}$. Finalmente, la semilla $\mathrm{F}_{2}$ se sembró en el ciclo Otoño-Invierno 2012/2013 en un lote de 700 $\mathrm{m}^{2}$ y se realizó la autofecundación de 250 plantas, elegidas al azar, para derivar las 193 líneas $S_{1}$ (población segregante y material genético de este estudio).

La evaluación de las 193 líneas $S_{1}$, progenitores y testigo se realizó bajo condiciones de riego y sequía inducida en prefloración (etapa vegetativa V12). Los ambientes de riego se realizaron durante los ciclos primavera-verano 2013 y otoño-invierno 2013/2014 en el campo experimental de la EESuX, localizado en Ayala, Morelos, dicha localidad se ubica a $18^{\circ} 44^{\prime}$ de latitud norte y $98^{\circ} 54^{\prime}$ de longitud oeste, con altitud de $1285 \mathrm{~m}, 912 \mathrm{~mm}$ de precipitación media anual y de clima tropical cálido sub-húmedo (INEGI-MORELOS, 2017). Mientras que el estrés hídrico se indujo de manera artificial al suspender el riego en la etapa vegetativa V12 (Ritchie et al., 2008). Un ambiente de sequía se realizó en Cd. Ayala, Morelos durante el ciclo otoño-invierno 2013/2014 y el segundo en Tepalcingo, Morelos ( $18^{\circ} 26^{\prime} \mathrm{LN}, 98^{\circ} 18^{\prime} \mathrm{LW}, 1100 \mathrm{msnm}$, $912 \mathrm{~mm}$ y clima cálido $\left(20.5^{\circ} \mathrm{C}\right)$, durante otoño-invierno 2014/2015. Cabe mencionar que en este ambiente, el riego se restableció después de 28 días de estrés artificial.

La siembra de los experimentos se estableció a una densidad poblacional de $55000 \mathrm{p} \mathrm{ha}^{-1}$ con distancia entre surcos y plantas a 0.8 y $0.25 \mathrm{~m}$, respectivamente; la dosis de fertilización fue de 170-80-00 en dos aplicaciones (el 50 $\%$ del nitrógeno $(\mathrm{N})$ y el $100 \%$ del fósforo, al momento de la siembra y el resto del $\mathrm{N}$ en etapa la V6) [Trujillo, 2009]. El suelo fue de tipo vertisol en ambos ambientes con estructura arcillo-limosa y pH de 5.5; el riego fue por goteo, excepto en un ambiente (con precipitación pluvial), el pH del agua fue de 7.2. Durante el periodo de evaluación, la temperatura y humedad relativa fluctuaron de 21.8 a $19.5^{\circ} \mathrm{C}$ y 78.1 a 74.7 $\%$, respectivamente (primavera-verano 2013); en tanto que para otoño-invierno 2013/2014 la temperatura osciló de 20.0 a $23.4^{\circ} \mathrm{C}$ y la humedad relativa varió 54.2 a $62.3 \%$. En otoño-invierno $2014 / 2015$ las temperaturas fluctuaron de 22.21 a $27.22^{\circ} \mathrm{C}$ y la humedad relativa osciló de 37.78 a 63.55 $\%$ (IMTA, 2015). El control de gallina ciega (Phyllophaga spp.) y gusano cogollero [Spodoptera frugiperda (J. E. Smith)] se realizó con Diazinón granulado ${ }^{\circledR}$ y Palgus ${ }^{\oplus}$ a dosis de $20 \mathrm{~kg}$ ha-1 y $75 \mathrm{~mL} \mathrm{ha}^{-1}$, respectivamente.

El diseño experimental que se utilizó fue un látice alfa de $14 \times 14$ con dos repeticiones. La unidad experimental la representó un surco de $5 \mathrm{~m}$ de largo para cada línea. Las variables de respuesta evaluadas fueron: floración masculina (FM) y femenina (FF), intervalo antesis-emergencia de estigmas (ASI), altura de planta (AP), altura de mazorca (AM) y rendimiento de grano ajustado al $15.5 \%$ de humedad (RG). Las variables medidas se probaron para normalidad 
y se encontró falta de normalidad en la variable ASI, por lo que se realizó a los datos de esta variable la transformación siguiente:

$$
A S I_{T}=\ln \sqrt{A S I+10}
$$

Los análisis de varianza, pruebas de rango múltiple (Tukey, 0.05) y correlación lineal de caracteres cuantitativos se realizaron con el paquete estadístico Statistical Analysis System (SAS) [SAS, 1999]. La estimación de la heredabilidad en sentido estrecho $\left(\hat{h}^{2}\right)$ se realizó con base en los componentes de varianza, estimados a partir de las esperanzas de cuadrados medios y se determinó a través de la ecuación 1 :

$$
\hat{h}^{2}=\frac{\sigma_{A}^{2}}{\sigma_{F}^{2}}
$$

Ecuación 1

Donde $\sigma_{A}^{2}$ y $\sigma_{F}^{2}$ son la varianza ambiental y fenotípica, respectivamente. Asimismo se determinaron los errores estándar para las heredabilidades $\left[E E\left(\hat{h}^{2}\right)\right]$ con base a las
ecuaciones 2 y 3 .

$$
\begin{aligned}
& E E\left(\hat{h}^{2}\right)=\frac{E E\left(\hat{\sigma}_{G}^{2}\right)}{\hat{\sigma}_{F}^{2}} \\
& E E\left(\hat{\sigma}_{G}\right)=\left[\left(\frac{2}{r^{2}}\right)\left[\frac{C M L^{2}}{(l-1)+2}+\frac{C M E^{2}}{G L_{\text {error }}+2}\right]\right]^{\frac{1}{2}}
\end{aligned}
$$

Ecuación 2

Ecuación 3

Donde $E E\left(\hat{\sigma}_{G}\right), r, l, C M L, C M E$ y $G L_{\text {error }}$ son el error estándar de la varianza genética, número de repeticiones, número de líneas, cuadrado medio de líneas, cuadrado medio del error y grados de libertad del error, respectixamente. Los intervalos de confianza $\left(I C_{h^{2}}\right)$ al $95 \%$ para la $h$ se determinaron con las ecuaciones 4 y 5 .

$$
\begin{aligned}
& I C_{h_{L I}}^{2}=1-\left[\left(M_{1} / M_{2}\right) / F_{\alpha / 2: g l 2, g l 1}\right]^{-1} \\
& I C_{h_{L S}}^{2}=1-\left[\left(M_{1} / M_{2}\right) F_{\alpha / 2: g l 2, g l 1}\right]^{-1}
\end{aligned}
$$

Ecuación 4

Ecuación 5

Donde: $M_{1}$ y $M_{2}, F_{\alpha}, g l 2, g l 1$ son el cuadrado medio de líneas, el cuadrado medio de la interacción línea $\times$ ambiente, valor de tablas de distribución $F$, grados de libertad, respectivamente (Knapp et al., 1985).

\section{RESULTADOS Y DISCUSIÓN}

\section{Variación fenotípica de caracteres cuantitativos bajo} riego y sequía inducida en $\mathrm{V} 12$

La variación del resultado de la caracterización fenotípica (fenotipeo) en los caracteres cuantitativos de la población segregante, progenitores y testigo con base a los ambientes contrastantes de humedad, se manifestó en el análisis de varianza combinado (Tabla 1), donde las fuentes de variación de ambientes $(\mathrm{A})$ y líneas $(\mathrm{L})$ mostraron diferencias estadísticas $(P \leq 0.05 y 0.01)$ en la totalidad de los caracteres cuantitativos. En tanto que en la fuente de interacción línea $\times$ ambiente $(L \times A)$, el análisis de varianza no detectó diferencias estadísticas para los caracteres de floración masculina y rendimiento de grano. Los coeficientes de variación fluctuaron entre 3.72 y $35.30 \%$, estos valores correspondieron a floración masculina y rendimiento de grano, respectivamente.

El mejoramiento para estrés hídrico en maíz se realiza con evaluaciones en ambientes contrastantes de humedad (Duvick, 2001; Tollenar y Lee, 2002); en este contexto, las diferencias estadísticas $(P \leq 0.01)$ de los caracteres cuantitativos entre los ambientes (A) [riego y sequía]; se debieron a los efectos de la disponibilidad de agua aprovechable contenida en el suelo durante la evaluación de las líneas segregantes. El ambiente de riego en todo momento se mantuvo con humedad aprovechable mientras que en sequía, el riego se suspendió en la etapa V12 (antes de la antesis).

El periodo de estrés hídrico duró 28 días, y dicho periodo de estrés hídrico impactó en los caracteres bajo estudio (Daryanto et al., 2016). Las diferencias estadísticas ( $P \leq 0.01$ ) observadas en la fuente de variación de líneas ( $L$ ) se deben a la presencia de variabilidad genética entre los genotipos de la población segregante, dicha variabilidad resultó por las combinaciones alélicas múltiples que se derivaron al avanzar el nivel de endogamia de la progenie $F_{1}$ (producto del cruzamiento inicial de Ac7643 y B39), existe correspondencia de estos resultados con los que encontraron otros investigadores al evaluar líneas segregantes en ambientes contrastantes de humedad (Ribaut et al., 1996; Agrama y Moussa, 1996).

Por otra parte, la presencia de interacción línea $x$ ambiente $(L \times A)$ en cuatro de los seis caracteres resultaron del tipo de interacción de diferencias en magnitud, las que se explican por el alto número de genotipos que constituyen a la población segregante y al efecto del impacto diferencial de los factores ambientales impredecibles, dicha interacción repercute en la expresión fenotípica de los genotipos (Fehr, 1993).

Con base a los resultados del análisis estadístico anterior y para interpretar los resultados del impacto del estrés hídrico en los caracteres cuantitativos de las líneas segregantes $S_{1}$, en la Tabla 2 se sintetiza la información del comportamiento diferencial de las líneas $S_{1}$ a través de los ambientes de riego y sequía inducida en la etapa vegetativa V12.

El promedio de rendimiento de grano en al ambiente de sequía fue de $0.75 \mathrm{t} \mathrm{ha}^{-1}$ mientras que en riego resultó de 1.26 ton $\mathrm{ha}^{-1}$; al analizar dichos promedios, se observó que existió una reducción del rendimiento de grano del $40 \%$ en sequía con relación al ambiente de riego, este comportamiento es similar al que reportaron otros investigadores al evaluar 185 familias $F_{6: 7}$ en riego y sequía (1.41 y $3.18 \mathrm{t} \mathrm{ha}^{-1}$, respectivamente, con una disminución en rendimiento de grano del $44 \%$ ) [Austin y Lee, 1998]; sus resultados son acordes a los que se reporta en este estudio. En otro trabajo de 
Tabla 1. Cuadrados medios del análisis combinado de las variables medidas bajo el sistema riego-sequía.

Table 1. Combined analysis mean squares of the quantitative traits measured under stress and non-stress hydric environments.

\begin{tabular}{|c|c|c|c|c|c|c|c|}
\hline FV & GL & FM & $\begin{array}{l}\text { FF } \\
\text { d }\end{array}$ & $\begin{array}{c}\text { ASI } \\
\text { (trans.)t }\end{array}$ & $\begin{array}{l}\text { AP } \\
\mathrm{cm}\end{array}$ & $\begin{array}{l}\mathrm{AM} \\
\mathrm{Cm}\end{array}$ & $\begin{array}{c}\text { RG } \\
\text { t ha-1 }^{-1}\end{array}$ \\
\hline$A$ & 1 & $3691.9^{* *}$ & $12408.7^{* *}$ & $3.021^{* *}$ & $256031.5^{* * *}$ & $31333.1^{* *}$ & $104.82^{* *}$ \\
\hline $\mathrm{R} / \mathrm{A}$ & 6 & $2603.2^{* *}$ & $3377.5^{* *}$ & $0.195^{* *}$ & $10532.4^{* *}$ & $3724.2^{* *}$ & $12.72^{* *}$ \\
\hline$B / A \times R$ & 104 & $9.9^{*}$ & $32.5^{* *}$ & $0.014^{* *}$ & $338.7^{* *}$ & $117.0^{* *}$ & $0.33^{* *}$ \\
\hline $\begin{array}{l}\text { Líneas } \\
\text { (L) }\end{array}$ & 195 & $88.4^{* *}$ & $93.1^{* *}$ & $0.012^{* *}$ & $1119.2^{* *}$ & $529.4^{* *}$ & $0.65^{* *}$ \\
\hline$L \times A$ & 195 & $6.7 \mathrm{NS}$ & $19.2^{* *}$ & $0.009^{* *}$ & $416.9^{* *}$ & $149.0^{* *}$ & $0.14 \mathrm{NS}$ \\
\hline Error & 1066 & 7.8 & 10.8 & 0.005 & 123.6 & 47.6 & 0.13 \\
\hline CV (\%) & & 3.7 & 4.2 & 5.5 & 7.3 & 11.9 & 35.3 \\
\hline Media & & 74.8 & 78.3 & 1.3 & 152.3 & 58.1 & 1.02 \\
\hline $\mathrm{R}^{2}$ & & 0.83 & 0.84 & 0.66 & 0.84 & 0.81 & 0.73 \\
\hline
\end{tabular}

$\mathrm{FV}=$ Fuente de variación; $\mathrm{GL}=$ Grados de libertad; FM = Floración masculina; FF = Floración femenina; $\mathrm{ASI}=$ Intervalo antesis-emergencia de estigmas; $\mathrm{AP}=$ Altura de planta; $\mathrm{AM}=$ Altura de mazorca; $\mathrm{A}=$ Ambientes, $\mathrm{R}=$ Repeticiones, $\mathrm{B}=$ Bloques, ${ }^{*},{ }^{*}$ Significativo al 0.05 y 0.01 de probabilidad, respectivamente; $\dagger=$ valores transformados por $\ln (A S I+10)^{0.5} ; \mathrm{RG}=$ Rendimiento de grano.

Tabla 2. Promedios y rangos de caracteres en líneas $\mathrm{S}_{1}$ bajo riego y sequía artificial.

Table 2. Means and ranks of traits in $\mathrm{S}_{1}$ inbred lines under stress and non-stress hydric environments.

\begin{tabular}{|c|c|c|c|c|c|c|}
\hline Estadístico & $\begin{array}{c}\text { RG } \\
\text { t ha-1 }\end{array}$ & FM & $\begin{array}{l}\text { FF } \\
\text { d }\end{array}$ & ASI & AP & $A M$ \\
\hline \multicolumn{7}{|c|}{ Sequía } \\
\hline$\mu_{P S}\left(S_{1}\right)$ & 0.75 & 76 & 81 & 5 & 139 & 53 \\
\hline$R_{P S}\left(S_{1}\right)$ & $0.27-1.77$ & $70-85$ & $72-91$ & $2-13$ & $108-172$ & $35-79$ \\
\hline$\mu(A c 7643)$ & 0.70 & 77 & 83 & 6 & 152 & 66 \\
\hline$\mu(B 39)$ & 1.26 & 74 & 79 & 5 & 145 & 56 \\
\hline$\mu(A c 7729)$ & 1.44 & 77 & 81 & 4 & 157 & 67 \\
\hline \multicolumn{7}{|c|}{ Riego } \\
\hline$\mu_{P S}\left(S_{1}\right)$ & 1.26 & 73 & 76 & 2 & 165 & 63 \\
\hline$R_{P S}\left(S_{1}\right)$ & $0.68-3.36$ & $66-81$ & $68-83$ & $1-5$ & $118-233$ & $38-94$ \\
\hline$\mu(A c 7643)$ & 1.47 & 79 & 82 & 3 & 181 & 70 \\
\hline$\mu(B 39)$ & 2.64 & 67 & 68 & 2 & 160 & 58 \\
\hline$\mu(A c 7729)$ & 2.27 & 76 & 79 & 3 & 173 & 66 \\
\hline $\begin{array}{l}\mathrm{RG}=\text { Rendimi } \\
\text { Intervalo antes } \\
\text { ca; } \mu_{P S}\left(S_{1}\right) \\
\text { fenotípica de la }\end{array}$ & $\begin{array}{l}\text { o de grano } \\
\text { mergencia } \\
\text { romedio d } \\
\text { blación se }\end{array}$ & $\begin{array}{l}\mathrm{FM}=\mathrm{Fl} \\
\text { de estig } \\
\text { la pobla } \\
\text { gregante }\end{array}$ & $\begin{array}{l}\text { ación n } \\
\text { las; AP } \\
\text { ión seg }\end{array}$ & $\begin{array}{l}\text { sculin } \\
\text { Altura } \\
\text { gante; }\end{array}$ & $\begin{array}{l}\text { Floración } \\
\text { tta; AP = A } \\
\left.f_{1}\right)=\text { Ran }\end{array}$ & $\begin{array}{l}\text { na; ASI = } \\
\text { e mazor- } \\
\text { xpresión }\end{array}$ \\
\hline
\end{tabular}


estrés hídrico en maíz tropical evaluaron familias $\mathrm{F}_{3}$ en riego y sequía (Ribaut et al., 1997), estos investigadores encontraron que el promedio de rendimiento de grano fue de 6.99 y 2.73 $\mathrm{t} \mathrm{ha-1}$ en riego y sequía, respectivamente, en consecuencia el rendimiento de grano disminuyó cerca del $60 \%$. Por otra parte, al evaluar 150 líneas $\mathrm{F}_{2: 3}$ se detectó una reducción del rendimiento de grano del $17 \%$ entre los ambientes contrastantes de humedad (Veldboom y Lee, 1996).

Se establece que la disminución del rendimiento de grano depende de la intensidad del estrés hídrico y su efecto se acentúa si el estrés coincide en la etapa de prefloración y durante el llenado de grano del maíz (Daryanto et al., 2016). La disminución del rendimiento de grano se debe a que el estrés hídrico afecta a caracteres secundarios o componentes del rendimiento de grano tales como: número de granos por mazorca, peso de granos por mazorca y peso de mazorca. Estos caracteres se pueden considerar como criterio de selección para tolerancia a sequía (Frova et al., 1999).

El promedio en la floración masculina (FM) de las líneas $S_{1}$, bajo sequía, se prolongó $3 \mathrm{~d}$ con relación al promedio del ambiente de riego. En cuanto a la media de la floración femenina (FF), esta manifestó un retraso de $5 \mathrm{~d}$ en la emergencia de estigmas de las líneas $\mathrm{S}_{1}$ que se sometieron a sequía con respecto a riego. La respuesta fenotípica, promedio de las líneas $\mathrm{S}_{1}$, en el intervalo de antesis-emergencia de estigmas (ASI) fue de 5 d en sequía, mientras que en riego el ASI promedio fue de dos días. El efecto de la sequía en la altura de planta redujo el carácter en un $16 \%$ con relación al ambiente de riego.

Uno de los objetivos en esta investigación fue determinar un grupo de líneas $S$, con rendimiento de grano alto y ASI corto en sequía, y con buen comportamiento agronómico en riego. En este sentido, a continuación se muestran las líneas selectas que mostraron los criterios prestablecidos en cada ambiente de humedad.

\section{Líneas selectas para rendimiento de grano alto y ASI corto en sequía}

En primer lugar, el rendimiento de grano de las 193 líneas $\mathrm{S}_{1}$ se ordenó de manera descendente; posteriormente, se aplicó el $20 \%$ de presión de selección en ambos extremos para categorizar a las líneas tolerantes y susceptibles a estrés hídrico. En la Tabla 3, sólo se muestran siete líneas tolerantes y tres susceptibles. Los promedios y rangos de expresión fenotípica de los caracteres cuantitativos refieren a las 39 líneas selectas para rendimiento de grano alto y bajo. El rango de expresión fenotípica para rendimiento de grano en grupo de líneas tolerantes resultó de 0.961 a $1.767 \mathrm{t} \mathrm{ha}^{-1}$, estos valores correspondieron a las líneas LUM-36 y LUM-76, respectivamente. La media de las líneas selectas tolerantes mostró un incremento del $64 \%$ con relación a la media de rendimiento de grano de las líneas susceptibles. El promedio de ASI de las líneas tolerantes resultó de 4 d mientras que para las susceptibles fue de $7 \mathrm{~d}$. En el grupo de líneas selectas como tolerantes se observó que para el rendimiento de grano existieron líneas superiores al promedio para este carácter
Tabla 3. Líneas tolerantes y susceptibles con base en el rendimiento de grano evaluadas bajo estrés hídrico inducido en la etapa V12.

Table 3. Tolerant and susceptible $S_{1}$ inbred lines of maize based on grain yield evaluated under hydric stress induced in V12 vegetative stage.

\begin{tabular}{|c|c|c|c|c|c|c|}
\hline \multirow[t]{2}{*}{ Línea } & RG & FM & $\mathrm{FF}$ & ASI & AP & AM \\
\hline & $\mathrm{tha}^{-1}$ & & $d$ & & \multicolumn{2}{|c|}{$\mathrm{cm}$} \\
\hline \multicolumn{7}{|c|}{ Tolerantes } \\
\hline LUM-76 & 1.77 & 76 & 78 & 2 & 166 & 56 \\
\hline LUM-80 & 1.69 & 71 & 75 & 3 & 166 & 67 \\
\hline LUM-73 & 1.63 & 76 & 79 & 3 & 167 & 63 \\
\hline LUM-124 & 1.48 & 78 & 84 & 6 & 145 & 59 \\
\hline LUM-81 & 1.44 & 77 & 81 & 5 & 146 & 56 \\
\hline LUM-74 & 1.43 & 76 & 78 & 2 & 150 & 40 \\
\hline LUM-40 & 1.43 & 73 & 79 & 5 & 158 & 71 \\
\hline$\mu_{L S T}\left(S_{1}\right)$ & 1.18 & 76 & 80 & 4 & 146 & 56 \\
\hline$R_{L S T}\left(S_{1}\right)$ & $0.961-1.767$ & $70-81$ & $74-85$ & $2-10$ & $121-172$ & $35-74$ \\
\hline \multicolumn{7}{|c|}{ Susceptibles } \\
\hline LUM-126 & 0.33 & 83 & 88 & 5 & 134 & 57 \\
\hline LUM-43 & 0.32 & 75 & 81 & 6 & 136 & 45 \\
\hline LUM-35 & 0.27 & 75 & 86 & 10 & 125 & 46 \\
\hline$\mu_{L S S}\left(S_{1}\right)$ & 0.427 & 76 & 83 & 7 & 136 & 53 \\
\hline$R_{L S S}\left(S_{1}\right)$ & $0.269-0.529$ & $\begin{array}{c}72- \\
83\end{array}$ & $76-88$ & $\begin{array}{l}3- \\
13\end{array}$ & $119-157$ & $\begin{array}{c}41- \\
65\end{array}$ \\
\hline$\mu(A c 7643)$ & 0.70 & 77 & 83 & 6 & 152 & 66 \\
\hline$\mu(B 39)$ & 1.26 & 74 & 79 & 5 & 145 & 56 \\
\hline$\mu(A c 7729)$ & 1.44 & 77 & 81 & 4 & 157 & 67 \\
\hline
\end{tabular}

$\mathrm{RG}=$ Rendimiento de grano; FM = Floración masculina; FF = Floración femenina; $\mathrm{ASI}=$ Intervalo antesis-emergencia de estigmas; $\mathrm{AP}=$ Altura de planta; $\mathrm{AM}=$ Altura de mazorca; $\mu_{\text {LSToS }}\left(S_{1}\right), R_{\text {LST oS }}\left(S_{1}\right)=$ Promedio y rango de 39 líneas selectas para estrés hídrico tolerantes o susceptibles, respectivamente.

(1.18 $\left.\mathrm{t} \mathrm{ha}^{-1}\right)$ que superan o igualan en rendimiento de grano de los progenitores (B39, Ac7643) y al testigo (Ac7729). Al agrupar las 193 líneas $S_{1}$ en veinte clases fenotípicas para los caracteres cuantitativos de rendimiento de grano y ASI, se observaron líneas segregantes que superaron a los valores promedio de los progenitores y testigo, esto se debió a la presencia de herencia o segregación transgresiva (Figura 1a y $1 b)$.

La presencia de la segregación transgresiva se reportó en otros estudios con líneas segregantes para rendimiento de grano en maíz bajo estrés hídrico (Agrama y Moussa, 1996), y para el ASI (Ribaut et al., 1996). Este efecto genético se presenta al cruzar progenitores contrastantes y endogámi- 

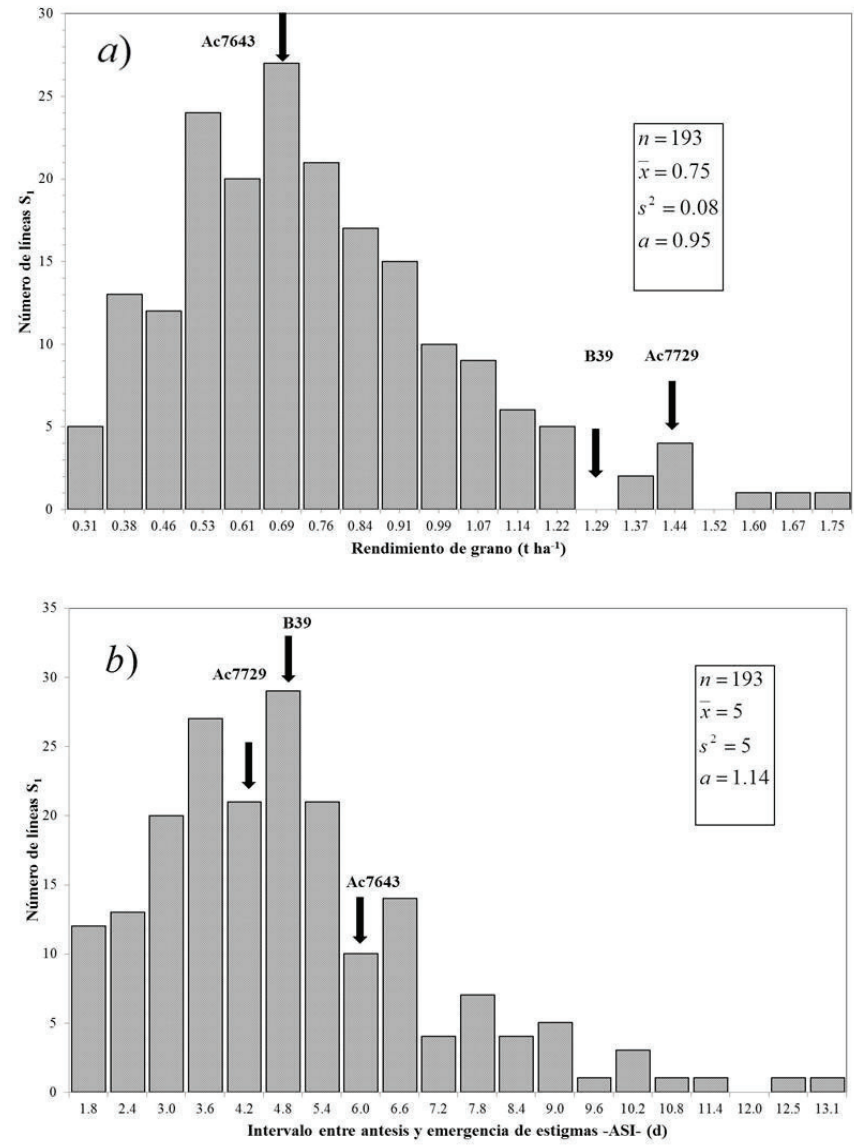

Figura 1. Frecuencias para los caracteres rendimiento de grano (a) e intervalo entre antesis y emergencia de estigmas-ASI (b) evaluados bajo estrés hídrico en la atapa vegetativa V12.

Figure 1. Grain yield (a) and ASI (b) traits frequencies evaluated under hydric stress in maize $V 12$ vegetative stage.

cos; desde el ámbito del mejoramiento genético, la aparición de genotipos con caracteres que superan a los progenitores posibilita tener germoplasma con características elegibles y deseables para su aprovechamiento. Tal es el caso de lo que se encontró en esta investigación, al cruzar las líneas endogámicas Ac7643 y $B 39$ y posteriormente avanzar la filial $F_{1}$ hasta el nivel $S_{1}$. Al someter a estrés hídrico a las líneas segregantes $S_{1}$, se activaron secuencias génicas que codificaron una respuesta fenotípica y se logró detectar líneas sobresalientes de rendimiento de grano alto y ASI corto; estas líneas se consideraron como tolerantes a dicho factor de tensión y son material genético base para generar una variedad sintética o híbridos tolerantes a estrés hídrico.

El rendimiento de grano del progenitor masculino (B39) en sequía fue de $1.26 \mathrm{t} \mathrm{ha}^{-1}$, superó a Ac7643 (progenitor femenino) y estuvo cerca de alcanzar al testigo (tolerante); por lo tanto la línea B39 presenta secuencias génicas que codifican a ASI corto y rendimiento de grano satisfactorio en sequía por lo que se tipifica como tolerante.

\section{Líneas selectas para rendimiento de grano en riego}

La Tabla 4 muestra siete líneas $S_{1}$ de un grupo de 39 líneas selectas con base a rendimiento de grano alto. El
Tabla 4. Líneas selectas con base en el rendimiento de grano en el ambiente de riego.

Table 4. Select lines based on grain yield evaluated under irrigation environment.

\begin{tabular}{lccccccc}
\hline Línea & RG & FM & FF & ASI & & AP & AM \\
\cline { 7 - 8 } & $\mathbf{t ~ h a - ~}^{-1}$ & & $\mathbf{d}$ & & & $\mathbf{c m}$ \\
\hline LUM-80 & 3.36 & 68 & 69 & 1 & & 233 & 87 \\
\hline LUM-18 & 2.10 & 70 & 72 & 2 & & 188 & 81 \\
\hline LUM-73 & 2.08 & 75 & 77 & 2 & & 199 & 86 \\
\hline LUM-97 & 2.00 & 69 & 72 & 3 & 180 & 72 \\
\hline LUM-21 & 1.97 & 69 & 71 & 2 & 194 & 84 \\
\hline LUM-87 & 1.95 & 69 & 70 & 1 & 183 & 67 \\
\hline LUM-51 & 1.92 & 67 & 69 & 1 & 151 & 50 \\
\hline$\mu_{L S R}\left(S_{1}\right)$ & 1.77 & 73 & 75 & 2 & 176 & 69 \\
\hline$R_{L S R}\left(S_{1}\right)$ & $1.56-3.36$ & $67-77$ & $69-82$ & $1-5$ & $118-233$ & $41-88$ \\
\hline$\mu(A c 7729)$ & 2.27 & 76 & 79 & 3 & 173 & 66 \\
\hline$\mu(A c 7643)$ & 1.47 & 79 & 82 & 3 & 181 & 70 \\
\hline$\mu(B 39)$ & 2.64 & 67 & 68 & 2 & 160 & 58 \\
\hline
\end{tabular}

$\mathrm{RG}=$ Rendimiento de grano; FM = Floración masculina; FF = Floración femenina; $\mathrm{ASI}=$ Intervalo antesis-emergencia de estigmas; AP = Altura de planta; $\mathrm{AM}=$ Altura de mazorca; $\mu_{L S R}\left(S_{1}\right)=$ Promedio de 39 líneas en riego; $R_{L S R}\left(S_{1}\right)=$ Rango de 39 líneas en riego.

promedio de las 39 líneas mostró un incremento del $40 \%$ con relación a la media de rendimiento de grano de las 193 líneas $\mathrm{S}_{1}$.

El objetivo principal de esta investigación se centra en la selección de líneas $S_{1}$ de rendimiento alto de grano y ASI corto en sequía y buen comportamiento agronómico en riego (Tabla 5).

La selección de las catorce líneas fue con base al carácter secundario del ASI bajo estrés hídrico, y se consideró a las líneas que presentaron hasta $3.3 \mathrm{~d}$ de ASI. El rendimiento de grano de estas líneas oscilo de 0.96 a $1.77 \mathrm{t} \mathrm{ha}^{-1}$ bajo estrés hídrico y en el ambiente de riego fue de 1.18 a $3.36 \mathrm{t} \mathrm{ha}^{-1}$. La selección de estas líneas fue con base a el comportamiento diferencial que las líneas presentaron a través de los ambientes de evaluación, estos resultados son acordes a los que se reportan en estudios de estrés hídrico (Duvick, 2001; Tollenar $y$ Lee, 2002).

El periodo de crecimiento agroclimático del maíz requiere de $150 \mathrm{~d}$ en los cuales deben existir de 500 a $800 \mathrm{~mm}$ de precipitación pluvial en zonas tropicales para completar su ciclo biológico; en este contexto, la caracterización agroclimática de las regiones o zonas productoras de este cereal, el evaluar el período de crecimiento agroclimático y con la obtención de germoplasma con regiones cromosómicas que confieren tolerancia a sequía se puede reducir los costos de 
Tabla 5. Líneas de ASI corto en sequía y buen rendimiento de grano en sequía y riego.

Table 5. Lines inbred witch short ASI and yield grain performance evaluated under irrigation and hydric stress environments.

\begin{tabular}{|c|c|c|c|}
\hline Línea & $\operatorname{ASI}^{1}(\mathrm{~d})$ & $\mathbf{R G}^{1}\left(\mathbf{t ~ h \mathbf { a } ^ { - 1 } )}\right.$ & $R^{2}\left(t h a^{-1}\right)$ \\
\hline LUM-188 & 1.8 & 1.35 & 1.69 \\
\hline LUM-120 & 1.8 & 1.05 & 1.33 \\
\hline LUM-185 & 1.8 & 0.99 & 1.33 \\
\hline LUM-76 & 2.0 & 1.77 & 1.86 \\
\hline LUM-74 & 2.0 & 1.43 & 1.43 \\
\hline LUM-123 & 2.3 & 0.97 & 1.87 \\
\hline LUM-73 & 2.5 & 1.63 & 2.08 \\
\hline LUM-79 & 2.8 & 1.18 & 1.39 \\
\hline LUM-174 & 2.8 & 1.18 & 1.62 \\
\hline LUM-46 & 2.8 & 1.09 & 1.35 \\
\hline LUM-12 & 2.8 & 0.96 & 1.55 \\
\hline LUM-102 & 3.0 & 1.00 & 1.58 \\
\hline LUM-80 & 3.3 & 1.69 & 3.36 \\
\hline LUM-98 & 3.3 & 1.08 & 1.18 \\
\hline $\mathrm{DSH}_{(0.05)}$ & 7.3 & 0.76 & 1.01 \\
\hline$\mu_{L T}\left(S_{1}\right)$ & 2.5 & 1.24 & 1.69 \\
\hline$R_{L T}\left(S_{1}\right)$ & $1.8-3.3$ & $0.96-1.77$ & $1.18-3.36$ \\
\hline
\end{tabular}

$\mathrm{ASI}^{1}=$ Intervalo antesis-emergencia de estigmas en sequía; $\mathrm{RG}^{1}, \mathrm{RG}^{2}=$ Rendimiento de grano en sequía y riego, respectivamente; $\mu_{L T}\left(S_{1}\right)=$ promedio de catorce líneas tolerantes a estrés hídrico y buen rendimiento de grano en riego; $R_{L T}\left(S_{1}\right)=$ Rango de catorce líneas tolerantes a estrés hídrico y buen rendimiento de grano en riego. producción y obtener un ahorro significativo del volumen de agua en el cultivo de maíz (Varas y Herrera, 2019).

Las estimaciones de la heredabilidad de los caracteres cuantitativos de la población segregante bajo estudio se presentan en el Tabla 6. De manera general, en el ambiente de riego se detectaron heredabilidades altas en la mayoría de los caracteres excepto en ASI y RG. Mientras que en el ambiente de sequía la mayoría de los caracteres resultaron con estimaciones de heredabilidad intermedias. La heredabilidad para rendimiento de grano resultó de $41 \%$, y fue menor a la estimación para el mismo carácter cuantitativo en condiciones de estrés intermedio $\left(h^{2}=0.66\right)$ en una población segregante de maíz (Ribaut et al., 1997). La heredabilidad que se estimó para la floración masculina (0.53) y para el ASI (0.41), en este estudio bajo sequía, difieren a los valores que se reportan con líneas segregantes de maíz tropical en la identificación de caracteres cuantitativos bajo estrés hídrico, los cuales fueron (0.86) y (0.78), para floración masculina y ASI, respectivamente (Ribaut et al., 1996).

El análisis de la asociación fenotípica entre caracteres, se muestra en el Tabla 7. En riego, sólo existió asociación estadística del rendimiento de grano con la altura de planta $\left(0.33^{* *}\right)$ y mazorca $\left(0.27^{* *}\right)$. Por otra parte, en el ambiente de sequía, se encontraron asociaciones negativas del rendimiento de grano con floración femenina $\left(-0.23^{* *}\right)$ y el ASI $\left(-0.37^{* *}\right)$, existe correspondencia de estos resultados con los que se reportan en la evaluación de familias $\mathrm{F}_{3}$ en el mapeo de QTLs para tolerancia a estrés hídrico en maíz en donde se encontró asociación negativa del rendimiento de grano bajo sequía con el ASI $\left(-0.449^{* *}\right)$ y la floración femenina $\left(-0.302^{* *}\right)$. Las correlaciones positivas del rendimiento de grano con la altura de planta $\left(0.21^{* *}\right)$ y mazorca $\left(0.41^{* *}\right)$ contrastan, en el mismo

Tabla 6. Heredabilidad de las variables bajo ambientes contrastantes de humedad. Table 6. Heritability of traits measured under contrasting humidity environments.

\begin{tabular}{|c|c|c|c|c|c|c|}
\hline \multirow[t]{2}{*}{ Parámetro } & FM & FF & ASI & AP & $A M$ & \multirow{2}{*}{$\begin{array}{c}\text { RG } \\
\text { t ha-1 }^{-1}\end{array}$} \\
\hline & \multicolumn{3}{|c|}{ d } & \multicolumn{2}{|c|}{$\mathrm{cm}$} & \\
\hline \multicolumn{7}{|c|}{ Riego } \\
\hline$h^{2}$ & $0.80 \pm 0.23$ & $0.81 \pm 0.23$ & $0.04 \pm 0.38$ & $0.97 \pm 0.19$ & $0.97 \pm 0.19$ & $0.42 \pm 0.34$ \\
\hline$I C h_{\text {inf. }}^{2}$ & 0.71 & 0.72 & -0.38 & 0.95 & 0.96 & 0.16 \\
\hline$I C h_{\text {sup. }}^{2}$ & 0.86 & 0.86 & 0.33 & 0.98 & 0.98 & 0.59 \\
\hline \multicolumn{7}{|c|}{ Sequía } \\
\hline$h^{2}$ & $0.53 \pm 0.20$ & $0.55 \pm 0.20$ & $0.41 \pm 0.21$ & $0.57 \pm 0.21$ & $0.64 \pm 0.21$ & $0.41 \pm 0.20$ \\
\hline$I C h_{\text {inf. }}^{2}$ & 0.33 & 0.36 & 0.15 & 0.39 & 0.48 & 0.16 \\
\hline$I C h_{\text {sup. }}^{2}$ & 0.67 & 0.69 & 0.59 & 0.70 & 0.75 & 0.59 \\
\hline
\end{tabular}

$\mathrm{h}^{2}$ = heredabilidad en sentido estrecho; FM = Floración masculina; FF = Floración femenina; $\mathrm{ASI}=$ Intervalo antesis-emergencia de estigmas; $\mathrm{AP}=$ Altura de planta; $\mathrm{AM}=$ Altura de mazorca; RG = Rendimiento de grano; $I C h^{2}{ }_{\text {Superior }}^{\text {Inferior }}=$ Intervalo de confianza para la heredabilidad $(a=0.05)$. 
Tabla 7. Coeficientes de correlación fenotípica entre caracteres en 193 líneas $\mathrm{S}_{1}$, progenitores y testigo bajo riego (debajo de diagonal) y sequía (arriba de diagonal).

Table 7. Phenotypic correlation coefficient between quantitative traits on $193 \mathrm{~S}_{1}$ inbred lines, parents and control line evaluated under irrigation (below the diagonal) and hydric stress (above the diagonal) environments.

\begin{tabular}{lcccccc}
\hline Carácter & FM & FF & ASI & AP & AM & RG \\
\hline FM & & $0.81^{* *}$ & $-0.11 \mathrm{NS}$ & $-0.05 \mathrm{NS}$ & $0.10 \mathrm{NS}$ & $-0.04 \mathrm{NS}$ \\
FF & $0.97^{* *}$ & & $0.48^{* *}$ & $-0.15^{*}$ & $.05 \mathrm{NS}$ & $-0.23^{* *}$ \\
ASI & $0.16 \mathrm{NS}$ & $0.36^{* *}$ & & $-0.19^{* *}$ & $0.08 \mathrm{NS}$ & $-0.37^{* *}$ \\
AP & $-0.19^{* *}$ & $-0.20^{* *}$ & $-0.06 \mathrm{NS}$ & & $0.76^{* *}$ & $0.41^{* *}$ \\
AM & $0.13 \mathrm{NS}$ & $0.11 \mathrm{NS}$ & $-0.03 \mathrm{NS}$ & $0.81^{* *}$ & & $0.21^{* *}$ \\
RG & $-0.12 \mathrm{NS}$ & $-0.13 \mathrm{NS}$ & $-0.12 \mathrm{NS}$ & $0.33^{* *}$ & $0.27^{* *}$ & \\
\hline
\end{tabular}

$\mathrm{RG}=$ Rendimiento de grano; $\mathrm{FM}=$ Floración masculina; $\mathrm{FF}=$ Floración femenina; $\mathrm{ASI}=$ Intervalo antesis-emergencia de estigmas; $\mathrm{AP}=$ Altura de planta; AM = Altura de mazorca; * ** Significativo y altamente significativo, respectivamente.

estudio (Agrama y Moussa, 1996) con las estimaciones de correlación fenotípica entre los mismos caracteres, las cuales resultaron no significativas.

En otro estudio reportaron la correlación positiva entre el ASI y las floración femenina $\left(0.63^{* *}\right)$ y resultó acorde con la que se reporta en este estudio, la cual fue de $0.48^{* *}$ (Ribaut et al., 1996).

\section{CONCLUSIONES}

El estudio de la expresión fenotípica de los caracteres cuantitativos en la población segregante de maíz a través de ambientes limitantes y no limitantes de humedad reveló que la floración es la etapa más sensible al estrés hídrico, una de las consecuencias morfológicas es el alargamiento del ASI y en función a la intensidad de la sequía se determinó la susceptibilidad o tolerancia de las líneas $\mathrm{S}$ de maíz. La herencia transgresiva en los caracteres para ASI y rendimiento de grano permitió seleccionar nuevos genotipos con caracteres agronómicos aprovechables y que mostraron tolerancia al estrés hídrico. El ASI corto es un carácter secundario y se correlaciona con el rendimiento de grano y se puede utilizar para realizar selección indirecta de genotipos tolerantes a través de este carácter en sequía, mientras que en riego no proporciona información. La herencia de la tolerancia a la sequía se puede utilizar para el desarrollo de híbridos o variedades sintéticas tolerantes a este factor de estrés. Por lo tanto, las líneas selectas bajo sequía pueden ser de utilidad en programas de mejoramiento para la obtención de germoplasma híbrido tolerante a estrés hídrico o la formación de una variedad sintética tolerante al mismo factor de tensión.

\section{REFERENCIAS}

Agrama, H. A. S. y Moussa, M. E. 1996. Mapping qtls in breeding for drought tolerance in maize (Zea mays L.). Euphytica. 91: 89-97.
Austin, D. F. y Lee, M. 1998. Detection of quantitative trait loci for grain yield and yield components in maize across generations in stress and nonstress environments. Crop Science. 38: 1296-1308.

Betrán, F. J., Ribaut, J. M., Beck, D. y González de León, D. 2003. Genetic diversity, specific combining ability, and heterosis in tropical maize under stress and nonstress environments. Crop Science. 43: 797-806.

Cairns, J. E., Crossa, J., Zaidi, P. H., Grudloyma, P., Sánchez C., Araus, J. L., Thaitad, S., Makumbi, D., Magorokosho, C., Bänziger, M., Menkir, A., Hearne, S. y Atlin, G. N. 2013. Identification of drought, heat, and combined drought and heat tolerant donors in maize. Crop Science. 53: 1335-1346.

Chen, J., Xu, W., Velten, J., Xin, Z. y Stout, J. 2012. Characterization of maize inbred lines for drought and heat tolerance. Journal of Soil and Water. 67: 354-364.

Daryanto, S., Wang, L. y Jacinthe, P. A. 2016. Global synthesis of drought effects on maize and wheat production. Plos One. 11 (5).

Duvick, D. N. 2001. Biotechnology in the 1930s: the development of hybrid maize. Nature Rev. of Genet. 2: 69-74.

Edmeades, G. O. 2013. Progress in achieving and delivering drought tolerance in maize - an update. ISAAA: Ithaca, NYisaaa.org.

Fehr, W. R. 1993. Principles of cultivar development. lowa State University Press V 1. Ames, lowa, USA.

Frova, C., Krajewski, P., Di Fonzo, N., Villa, M. y Sari-Gorla, M. 1999. Genetic analysis of drought tolerance in maize by molecular markers. I. Yield components. Theor Appl Genet. 99: 280-288.

González, S., Córdova, H., Rodríguez, S., De León, H. y Serrato, V. M. 1997. Determinación de un patrón heterótico a partir de la evaluación de un dialelo de diez líneas de maíz subtropical. Agronomía Mesoamericana. 8 (1): 01-07.

Instituto Mexicano de Tecnología del Agua (IMTA). 2015. Red de estaciones meteorológicas del estado de Morelos. Versión en CD.

Instituto Nacional de Estadística y Geografía (INEGI). 2017. Anuario Estadístico y Geográfico de Morelos. 502. Aguascalientes, Aguascalientes, México.

Kebede, A. Z., Melchinger, A. E., Cairns, J. E., Araus, J. L., Makumbi, D. y Atlin, G. N. 2013. Relationship of line per se and testcross performance for grain yield of tropical maize in drought and well-watered trials. Crop Science Society of America, Inc. 53 (4): 1228-1236.

Knapp, S. J., Stroup, W. W. y Ross, W. M. 1985. Exact confidence intervals for heritability on a progeny means basis. Crop Science. 25: 192-194.

Machado, F. O. D, Magalhães, P. C., De Oliveira, A. C., Dos Santos, M. X., Gomes, G. E. E. y Teixeira, G. C. 2002. Combining ability of tropical maize inbred lines under drought stress conditions. Crop Breeding. 6: 54-64.

Messina, C. D., Sinclair, T. R., Hammer, G. L., Curan, D., Thompson, J., Oler, Z., Gho, C. y Cooper, M. 2015. Limited-transpiration trait may increase maize drought tolerance in the US corn belt. Agronomy Journal. 107: 1978-1986.

Ramírez, D. J. L., Ron, P. J., Maya, L. J. B. y Cota, A. 1995. H-357 y H358. Híbridos de maíz de cruza simple para la zona subtropical y tropical de México. Folleto Técnico No. 4. Campo Experimental Centro de Jalisco, CIPAC-INIFAP. Tlajomulco, Jalisco, México. 20 p. 
Ribaut, J. M., Hoisington, D. A., Deutsch, V. C., Jiang, D. y González de León, D. 1996. Identification of quantitative trait loci under drought conditions in tropical maize. 1. Flowering parameters and the anthesis-silking interval. Theor Appl Genet. 94: 887-896.

Ribaut, J. M., Jiang, V., González de León, D., Edmeades, G. O. y Hoisington, D. A. 1997. Identification of quantitative trait loci under drought conditions in tropical maize. 2. Yield components and marker-assisted selection strategies. Theor Appl Genet. 94: 887-896.

Ritchie, S. W., Hanway, J. J. y Benson, O. G. 2008. Como se desarrolla una planta de maíz. Inpofos cono sur. Reporte especial No. 48: 08-10. Universidad de Ciencia y Tecnología del Estado de IOWA. Servicio cooperativo de extensión, Ames, IOWA.

Secretaría de Agricultura, Ganadería, Desarrollo Rural, Pesca y Alimentación (SAGARPA). 2018. Servicio de información estadística agroalimentaria y pesquera. Subsistema de Información Agrícola. Versión en CD.

Statistical Analysis System Institute (SAS). 1999. SAS user's guide. Statistics. Version 8. 2. SAS Inst. Cary, N. C.

Terrón, A., Preciado, E., Córdova, H., Mickelson, H. y López, R. 1997. Determinación del patrón heterótico de 30 líneas de maíz derivadas de la población 43sr del CIMMYT. Agronomía Mesoamericana. 8 (1): 26-34.
Tollenar, M. E. y Lee, A. 2002. Yield potential, yield stability and stress tolerance in maize. Field Crops Res. 75: 161-169.

Trujillo, C. A. 2009. Guía para cultivar maíz bajo condiciones de riego en el estado de Morelos. Instituto Nacional de Investigaciones Forestales Agrícolas y Pecuarias. Campo Experimental Zacatepec. Morelos. México.

Varas, M. C. y Herrera, S. M. 2019. Caracterización del periodo de crecimiento agroclimático del maíz (Zea mays) en la provincia los ríos. Revista de Ciencias Biológicas y de la Salud Biotecnia. 21 (1): 54-59.

Veldboom, R. y Lee, M. 1996. Genetic mapping of quantitative trait loci in maize in stress and nonstress environments: I. Grain yield and yield components. Crop Science. 36: 13101319.

Walsh, B. 2001. Quantitative Genetics. Encyclopedia of Life Sciences. Nature Publishing Group. 1-7.

Xue, Y., Warburton, M. L., Sawkins, M., Zhang, X., Setter, T., Xu, Y., Grudloyma, P., Gethi, J., Ribaut, J. M., Li, W., Zhang, X., Zheng, Y. y Yan, J. 2013. Genome-wide association analysis for nine agronomic traits in maize under well-watered and waterstressed conditions. Theoretical and Applied Genetics. 126 (10): 2587-2596. 